\title{
Rhodococcus induced false-positive galactomannan (GM), a biomarker of fungal presentation, in patients with peritoneal dialysis: case reports
}

Tamonwan Chamroensakchai ${ }^{1,2}$, Wasin Manuprasert ${ }^{2}$, Asada Leelahavanichkul ${ }^{1}$, Kullaya Takkavatakarn³, Nisa Thongbor ${ }^{4}$, Bunpring Jaroenpattrawut ${ }^{5}$ and Talerngsak Kanjanabuch ${ }^{2,3,6^{*}}$ [D

\begin{abstract}
Background: Galactomannan index (GMI) at a level higher than 0.5 provides high sensitivity and specificity for the diagnosis of fungal peritonitis. Here, we report the false-positive of GMI in peritoneal dialysis (PD) effluent (PDE) due to Rhodococcus peritonitis in PD patients.

Case presentation: GMI in PDE of case \#1 and case \#2 were 1.53 and 0.76 , respectively, while serum GMI of both cases was less than 0.5. In addition, GMl from the specimens obtained directly from the stationary phase of Rhodococcus colonies were 1.27 and 1.56, which were isolated from case \#1 and \#2, accordingly.

Conclusion: High GMI in PDE of PD patients is not specific just for fungal infections but may also be secondary to other infections, such as Rhodococcus spp., especially in endemic areas.
\end{abstract}

\section{Background}

Fungal peritonitis is a relatively uncommon but serious complication of peritoneal dialysis (PD) and is associated with a high risk of technique failure and mortality, especially in cases of delayed diagnosis. The galactomannan (GM) test is an easier technique for the detection of fungal cell wall components shedding out of the fungi during fungal growth and death [1]. Indeed, GM index (GMI), analyzed by a sandwich-ELISA (Bio-Rad Platelia ${ }^{\mathrm{Tm}}$, USA), has been widely used for the diagnosis of systemic fungal infection [2] and has been recently proposed as an alternative marker of fungal peritonitis [3]. Despite the high sensitivity and specificity of GMI in PD effluent (PDE) for the diagnosis of fungal peritonitis [3] and fungal colonization inside a PD catheter [4], we reported a false-positive GMI in PDE from patients infected with Rhodococcus spp. There were 2 patients with rhodococcal infection who had

\footnotetext{
*Correspondence: golfnephro@hotmail.com

${ }^{2}$ Center of Excellence in Kidney Metabolic Disorders, Faculty of Medicine,

Chulalongkorn University, Bangkok, Thailand

${ }^{3}$ Division of Nephrology, Department of Medicine, Faculty of Medicine,

Chulalongkorn University, Bangkok, Thailand

Full list of author information is available at the end of the article
}

orange stains inside their PD catheters. Both cases were mistakenly diagnosed as fungal peritonitis and resulting in initial treatment failure.

\section{Case presentation}

Case \#1 was a 71 year-old man with diabetic end-stage renal disease (ESRD) who was performing continuous ambulatory PD (CAPD) at Sunpasitthiprasong Hospital since 2013. On 30 July 2017 (day 0), he presented with acute abdominal pain and cloudy dialysate associated with a normal exit-site. The diagnosis of peritonitis was confirmed with a PDE leukocyte count of 2900 cells $/ \mu \mathrm{L}$ of which $91 \%$ were neutrophils. The empirical therapy for bacterial peritonitis with intraperitoneal (IP) cefazolin $1 \mathrm{~g}$ together with ceftazidime $1 \mathrm{~g}$ IP once daily was commenced. Although the dialysate leukocyte gradually decreased, the leukocyte persisted at more than $100 / \mu \mathrm{L}$ with neutrophil predominance. The dialysate from day 0 failed to culture any organism. However, many orange spots were noticed inside the PD catheter on day +8 . GMI in the PDE and the sera were 1.53 (normal $<0.5$ ) and 0.39 (normal<0.5), respectively. The provisional diagnosis of fungal peritonitis was made. Intravenous 
amphotericin $\mathrm{B}, 0.5 \mathrm{mg} / \mathrm{kg} /$ day was promptly started on the following day $($ day +9$)$, and the PD catheter was subsequently removed on day +11 . The PD catheter, serum, and drained PDE were submitted to the central microbiology lab for microorganism identification. The patient was transferred to hemodialysis (HD) following PD catheter removal. The patient continued to improve while having intravenous (IV) amphotericin and ceftazidime that were prescribed for a total of 2 weeks.

Case \#2, a 59 year-old man with a past medical history of diabetes and hypertension, was diagnosed with ESRD from diabetes. He commenced CAPD (4 exchanges/day) in January 2017 at Nakhon Pathom Hospital. While performing CAPD, he was able to maintain adequate dialysis small solute clearance and had never experienced peritonitis. On 16 January 2018 (day 0) the patient presented with cloudy dialysate, abdominal pain, poor appetite, and ultrafiltration failure, associated with a normal exit-site. The PDE cell count was 497 cells $/ \mu \mathrm{L}$ with a neutrophil predominance (63\%). He was initially diagnosed with CAPD-related peritonitis and received a combination of IP cefazolin and ceftazidime on the same day resulting in partial resolution of the abdominal pain. However, the PDE leukocyte count that was repeated on day +3 indicated an increased count of 2080 cells $/ \mu \mathrm{L}$ ( $85 \%$ neutrophils), while the dialysate culture from day 0 later yielded Corynebacterium spp. The primary physician swapped the antibiotics to IV vancomycin, $1 \mathrm{~g}$ every 3 days, and IP amikacin, $25 \mathrm{mg}$ loading dose followed by $12 \mathrm{mg}$ once daily for a total duration of 2 weeks. During the follow-up period, salmon-pink colonies were observed inside the PD catheter and GMI in PDE from day +10 was later reported positive at a level of $0.76(<0.5)$. Superimposed fungal peritonitis was suspected, although IP amphotericin B was added on day + 13 , and the PD catheter was removed on day +16 . Amphotericin was later withdrawn after a return of culture report from the central microbiology lab on day + 19 demonstrated Rhodococcus equi. Temporary HD was performed during day +17 to day +56 . The PD catheter was reinserted on day +58 , and the patient has remained well on CAPD without any subsequent peritonitis.

At the central microbiology lab, the removed catheters and the PDE were submitted for culture in several media. Interestingly, the orange colonies on tryptic soy agar (TSA) and chocolate agar were observed at $72 \mathrm{~h}$, but no colony growth on SDA (Fig. 1). Rhodococcus spp. and Rhodococcus equi was identified by modified AFB and the biochemistry assay from case \#1 and \#2, respectively. The GMI in the specimens obtained directly from colonies of Rhodococcus were 1.27 (Case \#1) and 1.58 (case \#2), which were above the cut-off value $(\geq 0.5)$ using a GM test kit (Bio-Rad Platelia ${ }^{\mathrm{TM}}$, USA).

\section{Discussion and conclusion}

Rhodococcus, a gram-positive filamentous bacteria which belongs to phylum Actinobacteria, causes disease only in immune-compromised hosts [5]. This organism can
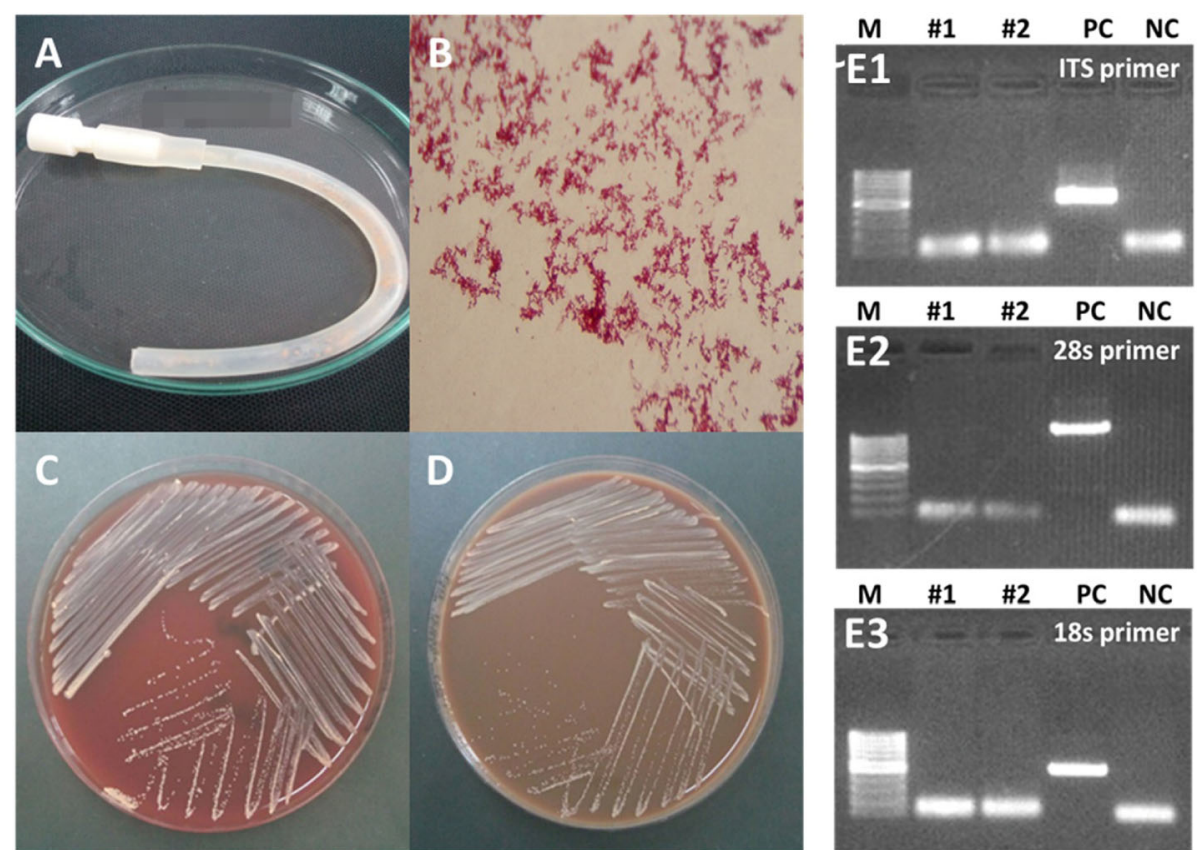

Fig. 1 The orange stains on (a) PD catheter. b Modified acid-fast stain of TS catheter specimen depicts numerous microorganisms. The orange colony was grown on (c) Blood agar, and (d) chocolate agar after $72 \mathrm{~h}$. Fungal co-infection was excluded by various fungal primers, including ITS (E1), $28 \mathrm{~S}$ (E2), and $18 \mathrm{~S}$ (E3) primers. Abbreviations: NC, negative control; PC, positive control 
survive in various habitats such as terrestrial (soil) and aquatic environments (fresh and saltwater) [6, 7]. Fungal co-infection was excluded from the presented cases using both fungal culture and PCR with universal fungal primers (16S, 28S, and ITS) (Fig. 1e). Although Rhodococcus is sensitive to several antibiotics [6], early catheter removal was performed in both cases because of the misdiagnosis of fungal peritonitis by the primary physicians. The ISPD Guideline 2016 recommends an immediate PD catheter removal when fungi are identified [8]. Thailand has a high incidence of culture-negative peritonitis, particularly in provincial hospitals. The falsepositive GMI caused by this infection has never been mentioned despite the reported lower sensitivity and specificity for the GM test (77 and 58\% respectively) [3].

The cell envelop of the Rhodococcus does not express GM, it contains a substantial amount of arabinogalactan [AG: a linear galactan of galactofuranose (Galf) residues + discrete arabinan side branches which contain arabinofuranoses) and lipoarabinomannan (LAM: lipoglycan core + mannan side chains) of which both are bound to branched long-chain lipids termed mycolic acids. The galactan of $R$. equi contains a mixture of $(1 \rightarrow 3),(1 \rightarrow 5)$, and $(1 \rightarrow 6)$ linkages [7]. On the other hand, GM found in pathologic filamentous fungi is a hetero-polysaccharide consisting of a linear-mannan core with a repeating tetramannose unit (2M-6 M-2 M-2 M) and its side chains (4-5 residues of $\beta-(1 \rightarrow 5)$-Galf attached to mannose) $[9,10]$. Both organisms contain $\beta-(1 \rightarrow 5)$-Galf which is a target for the immune system and monoclonal antibody using in the commercial GM test kits (Bio-Rad Platelia ${ }^{\mathrm{ma}}$, USA and Pastorex Aspergillus ${ }^{\mathrm{Tn}}$, Diagnostic Pasteur, France) $[9,10]$. However, the Galf is located in a different strand of carbohydrate heteropolymer where filamentous mold is on the side chain of GM while the Rhodococcus is on the backbone of AG [9].

Since there are very few case reports of rhodococcal peritonitis, experiences of the preferred treatment are limited. A combination of antibiotics is the mainstay of treatment, especially antibiotics with intracellular activity against the organism. Although Rhodococcus is initially sensitive to cephalosporins, it is not recommended in clinical practice because of the potential development of late resistance [11]. The duration of treatment in immunocompetent patients may range from 2 to 8 weeks or even longer depending upon the severity of the disease [12]. Removal of the PD catheter should be considered in Rhodococcus peritonitis due to the unpredictable response of this organism to treatment [13]. Our 2 cases demonstrated the success of management of Rhodococcus peritonitis with 2 weeks of antibiotics combined with the PD catheter removal. Case \#2 was treated with IP vancomycin plus amikacin. Meanwhile, case \#1 was successfully treated by IP ceftazidime. The Rhodococcus infections in both of these cases either responded to the empirical treatment or PD catheter removal.

In conclusion, false-positive GMI results may occur with Rhodococcus infection. Thus, various culture media and polymerase chain reaction should be used to confirm the results of high GMI in PDE, especially in endemic areas of rhodococcal infection.

\begin{abstract}
Abbreviations
AFB: Acid-fast stain; AG: Arabinogalactan; CAPD: Continuous ambulatory peritoneal dialysis; ESRD: End-stage renal disease; GM: Galactomannan; GMI: Galactomannan index; HD: Hemodialysis; IP: Intraperitoneal; ISPD: International Society for Peritoneal Dialysis; IV: Intravenous; LAM: Lipoarabinomannan; PCR: Polymerase chain reaction; PD: Peritoneal dialysis; PDE: Peritoneal dialysis effluent; SDA: Sabouraud dextrose agar; TSA: Tryptic soy agar
\end{abstract}

\section{Acknowledgments}

We are special thanks to Ms. Margaret Clarke for her language guidance and Neil Boudville, M.D., Medical School, University of Western Australia, Perth, Australia for full language support.

\section{Authors' contributions}

TC and TK contributed to the study design. WM, NT, and BJ collected data during the study. TC, WM, AL, KT, and TK developed the first draft of the manuscript which was then reviewed and intensively revised by the other authors. TK and AL revised accordingly and responded to the reviewers. All authors read and approved the final manuscript.

Funding

This study is supported by the Ratchadaphiseksomphot Endorsement Fund (CU-GRS_60_12_30_05), Chulalongkorn University, Thailand, The National Research Council of Thailand (156/2560), and Thailand Research Foundation (TRF), Thailand (IRG5780017).

\section{Availability of data and materials}

The data that support the findings of this study are available from the corresponding author upon reasonable request.

Ethics approval and consent to participate

Not applicable.

Consent for publication

The patient provided written informed consent to this case's publication.

\section{Competing interests}

The authors declare that they have no competing interests.

\section{Author details}

${ }^{1}$ Department of Microbiology, Faculty of Medicine, Chulalongkorn University, Bangkok, Thailand. ${ }^{2}$ Center of Excellence in Kidney Metabolic Disorders, Faculty of Medicine, Chulalongkorn University, Bangkok, Thailand. ${ }^{3}$ Division of Nephrology, Department of Medicine, Faculty of Medicine, Chulalongkorn University, Bangkok, Thailand. ${ }^{4}$ Department of Medicine, Sunpasitthiprasong Hosptial, Ubon Ratchathani, Thailand. ${ }^{5}$ Continuous Ambulatory Peritoneal Dialysis Clinic (CAPD), Nakhon Pathom Hospital, Nakhon Pathom, Thailand. ${ }^{6}$ CAPD Excellent Center, King Chulalongkorn Memorial Hospital, Bangkok, Thailand.

Received: 19 March 2019 Accepted: 25 November 2019

Published online: 02 December 2019

\section{References}

1. Wingard JR. Have novel serum markers supplanted tissue diagnosis for invasive fungal infections in acute leukemia and transplantation? Best Pract Res Clin Haematol. 2012;25(4):487-91.

2. Hachem RY, Kontoyiannis DP, Chemaly RF, Jiang Y, Reitzel R, Raad I. Utility of galactomannan enzyme immunoassay and $(1,3) \beta$-D-Glucan in diagnosis of invasive fungal infections: low sensitivity for aspergillus fumigatus 
infection in hematologic malignancy patients. J Clin Microbiol. 2009;47(1): 129-33.

3. Worasilchai N, Leelahavanichkul A, Kanjanabuch T, Thongbor N, Lorvinitnun $P$, Sukhontasing $K$, et al. (1 $\rightarrow 3)-\beta$-D-glucan and galactomannan testing for the diagnosis of fungal peritonitis in peritoneal dialysis patients, a pilot study. Med Mycol. 2015;53(4):338-46.

4. Leelahavanichkul A, Pongpirul K, Thongbor N, Worasilchai N, Petphuak K, Thongsawang B, et al. (1 $\rightarrow 3)-\beta$-D-Glucan and Galactomannan for a differentiation of chemical "black particles" and fungal particles inside peritoneal Dialysis tubing. Perit Dial Int. 2015;36:402-9.

5. Navarro-Llorens JM, Patrauchan MA, Stewart GR, Davies JE, Eltis LD, Mohn WW. Phenylacetate catabolism in Rhodococcus sp. strain RHA1: a central pathway for degradation of aromatic compounds. J. Bacteriol. 2005;187(13): 4497-504.

6. Riesenberg A, Feßler AT, Erol E, Prenger-Berninghoff E, Stamm I, Böse R, et al. MICs of 32 antimicrobial agents for Rhodococcus equi isolates of animal origin. J Antimicrob Chemother. 2014;69(4):1045-9.

7. Daffe M, McNeil M, Brennan PJ. Major structural features of the cell wall arabinogalactans of Mycobacterium, Rhodococcus, and Nocardia spp. Carbohydr Res. 1993;249(2):383-98.

8. Li PK, Szeto CC, Piraino B, de Arteaga J, Fan S, Figueiredo AE, et al. ISPD peritonitis recommendations: 2016 update on prevention and treatment. Perit Dial Int. 2016;36(5):481-508.

9. Stynen D, Sarfati J, Goris A, Prevost MC, Lesourd M, Kamphuis H, et al. Rat monoclonal antibodies against Aspergillus galactomannan. Infect Immun. 1992;60(6):2237-45.

10. Van Cutsem J, Meulemans L, Van Gerven F, Stynen D. Detection of circulating galactomannan by Pastorex Aspergillus in experimental invasive aspergillosis. Mycoses. 1990;33(2):61-9.

11. Giguere S, Lee EA, Guldbech KM, Berghaus LJ. In vitro synergy, pharmacodynamics, and postantibiotic effect of 11 antimicrobial agents against Rhodococcus equi. Vet Microbiol. 2012;160(1-2):207-13.

12. Kedlaya I, Ing MB, Wong SS. Rhodococcus equi infections in immunocompetent hosts: case report and review. Clin Infect Dis. 2001;32(3): E39-46.

13. Tang S, Lo CY, Lo WK, Ho M, Cheng IK. Rhodococcus peritonitis in continuous ambulatory peritoneal dialysis. Nephrol Dial Transplant. 1996; 11(1):201-2.

\section{Publisher's Note}

Springer Nature remains neutral with regard to jurisdictional claims in published maps and institutional affiliations.

Ready to submit your research? Choose BMC and benefit from:

- fast, convenient online submission

- thorough peer review by experienced researchers in your field

- rapid publication on acceptance

- support for research data, including large and complex data types

- gold Open Access which fosters wider collaboration and increased citations

- maximum visibility for your research: over $100 \mathrm{M}$ website views per year

At $\mathrm{BMC}$, research is always in progress.

Learn more biomedcentral.com/submissions 